\title{
Incidencia de los entornos virtuales de aprendizaje en la calidad de la educación superior, desde el contexto colombiano'
}

\author{
Karolina González Guerrero ${ }^{2}$ \\ Norma Costanza Berdugo ${ }^{3}$ \\ Adriana Mortigo Rubio ${ }^{4}$
}

Recibido: 18-07-2017

Aceptado: 27-10-2017

\section{RESUMEN}

Los procesos de globalización y estandarización en el contexto de educación superior, han traído un referente de implementación y uso de las Tecnologías de la Información y la Comunicación (TIC), como medio para la formación en la educación superior, en la actual sociedad de la información. No obstante, este hecho demanda un análisis crítico acerca de la calidad que se ofrece en este contexto virtual y, en especial, la modalidad concerniente al aprendizaje mixto. Para ello, el estudio muestra una metodología fundamentada, consecuente con la revisión documental y el análisis comparativo de literatura, capaz de establecer un marco de análisis de las implicaciones del b-learning en el contexto de la educación superior. De este modo, la discusión se basa en las relaciones de uso pedagógico de las herramientas y entornos virtuales, en pro de apoyar la formación presencial y, a la par, sustentar un desarrollo educativo de calidad en instituciones de educación superior. A manera de conclusión,

1. Articulo resultado el proyecto de investigación ING 2478 - 2017 Financiado por la Vicerrectoría de investigaciones de la Universidad Militar Nueva Granada.

2. L Licenciada en electrónica, Magíster en Educación, Doctora en Educación, docente - investigadora Universidad Militar Nueva Granada.

Correo electronico: karolina.gonzalez@unimilitar.edu.co ORCID: 0000-0002-9762-579X

3. Trabajadora Social, Especialista en docencia universitaria, especialista en mercado y servicios y magister en gestión de organizaciones. Docente investigadora Universidad Militar Nueva Granada.

Correo electronico: norma.berdugo@unimilitar.edu.co

ORCID: 0000-0001-7024-704X

4. Licenciada en educación, Especialista en docencia universitaria, especialista en mercado y servicios y magister en gestión de organizaciones. Docente investigadora Universidad Militar Nueva Granada.

Correo electronico: adriana.mortigo@unimilitar.edu.co 
se plantea una necesidad de fortalecer los procesos pedagógicos que soportan los recursos utilizados en el b-learning, en tanto se propicie una calidad educativa en términos de eficiencia, pertinencia y la adquisición de competencias para el mundo de la vida.

Palabras clave: b-learning, calidad educativa, educación superior, evaluación docente, herramientas virtuales.

\title{
Incidence of Virtual Learning Environments in Higher Education of Quality from the Colombian context
}

\begin{abstract}
The processes of globalization and standardization in the context of higher education, have brought a reference implementation and use of information and communications technology as a means to form in today's information society. However, this fact demands a critical analysis of the quality offered in this virtual context and, in particular, concerning the blended learning mode. To do so, the study shows a consistent methodology based document review and comparative analysis of literature, able to establish a framework for analysis of the implications of b-learning in the context of higher education. Thus, the discussion is based on the relationships of pedagogical use of tools and virtual environments towards supporting classroom training and, at par, support educational development of quality in higher education institutions. In conclusion, arises a need to strengthen the pedagogical processes that support the resources used in the b-learning, as an educational quality in terms of efficiency, relevance and the acquisition of skills for the world of life is promoted.
\end{abstract}

Keywords: b -learning, quality of education, higher education, teacher evaluation, virtual tools. 


\section{Introducción}

Las universidades como epicentro de transformaciones culturales, acercan el conocimiento al sujeto sin fronteras, participando de un cambio social, en tanto que el núcleo de personas educadas por la institución forme parte de una comunidad de transformación. No obstante, González y León (2010) aclaran que la calidad educativa en instituciones universitarias ha disminuido, conforme los recursos otorgados a la formación docente, la investigación e innovación, no son suficientes para el favorecimiento de comunidades educativas. Por otro lado, se mencionan los graves problemas de desigualdad de progreso en las sociedades, esto implica las pocas oportunidades que tienen las personas de bajos recursos para acceder a una formación de orden superior, pues los procesos de globalización, si bien han roto las barreras entre sociedades, también han generado una crítica a la falta de espacios y oportunidades educativas. Estas problemáticas hacen coherente pensar en impulsar sistemas dinámicos de formación, los cuales en palabras de Regnet y Martínez (2010), abren una puerta a la necesidad educativa de formar con calidad; una opción de fuerte acogida, se basa en los espacios mixtos de formación: b-learning.

En el presente estudio se refleja el análisis reflexivo de los fundamentos que sustentan los entornos virtuales y su contribución a la calidad educativa, desde tres ejes temáticos principales; el primero, concerniente al b-learning (Guarneros, Silva \& Pérez, 2009; González, 2007; Gros \& Silva, 2005; Fainholc, 2008) como epicentro de inclusión de los entornos virtuales para potencializar la labor presencial de los docentes; como segundo eje, se expone la necesidad de articular un proceso pedagógico en los recursos y herramientas virtuales incluidas dentro de los entornos virtuales, a partir de los postulados de De Benito \& Salinas, 2003; Marcelo, 2005; y Pérez \& Mestre, 2007, y por último, se establece una finalidad de inclusión de dichos entornos virtuales a través del b-learning como un medio de aporte a la calidad en la educación superior según Padilla (2008); González, Padilla y Rincón (2011).

De otro lado, se hace uso de una metodología encaminada a establecer una emergencia en las fuentes documentales, las cuales a la luz de la teoría fundamentada (Strauss y Corbin, 2002), develan la importancia de explicar y contextualizar un fenómeno ya estudiado -como lo es el b-learning- y su incursión en la educación superior, teniendo en cuenta 
la búsqueda y selección de información, el proceso de codificación de los documentos a través del Software Atlas ti.

El artículo deja entrever, como conclusiones, la necesidad de estudios exhaustivos acerca de los roles, competencias y formas de evaluar al docente; cuyo perfil, funciones y contribuciones dan cabida a mejorar la implementación de entornos virtuales de aprendizaje en el mundo globalizado, que, si bien se muestra somero en cuanto a incentivar la equidad social, es un escenario propenso a la transformación social por medio de la educación al servicio de un desarrollo socio-cultural.

\section{Concepción del b-learning en la educación superior}

El blended learning o b-learning es una modalidad mixta de formación que establece la combinación de la instrucción presencial y los elementos comunicativos, metodológicos y tecnológicos de los ambientes virtuales. Este sistema combinado de formación trata de adoptar las mejores condiciones de cada entorno, a la par de suplir las falencias que posee respectivamente dichos espacios. Regnet y Martínez (2010) describen el blended learning como una posibilidad de oferta educativa, que actualmente muestra una tendencia al crecimiento por las bondades ofrecidas a la hora de articular diversos procesos y estrategias, favoreciendo los estilos de aprendizaje del estudiante. De igual forma, se plantea un cambio paradigmático en los procesos pedagógicos y en los roles del docente, al surgir nuevas funciones y desempeños congruentes con los escenarios virtuales y presenciales de formación.

En los entornos virtuales, se plantea como alternativa el uso de la informática e internet para efectuar mediaciones pedagógicas, didácticas y comunicativas, solucionando problemas de interacción y accesibilidad al proceso formativo (Sánchez, 2008). Este hecho hace prioritario el diseño y uso de interfaces eficientes, con el fin de dar respuesta a las demandas particulares de cada área disciplinar (Gros \& Silva, 2005), por ende se debe hacer evidente una estructura cooperativa, flexible, sistémica y multidisciplinaria, asumiendo el desafío divergente de articular orientaciones pedagógicas y teorías que den respuesta efectiva a estos cambios sociales implicados directamente en las formas como se aprende y educa en la actualidad. 
Desde los espacios presenciales las sesiones se encaminan a la orientación personalizada o grupal, relacionada con las prácticas, talleres y debates sobre hechos actuales o discusiones que ameritan interacciones receptivas o emotivas a partir de dinámicas diferentes a las desarrolladas en los medios virtuales (Gros \& Silva, 2005). El hecho de pertenecer a una comunidad educativa reaviva el fundamento presencial (Pérez \& Mestre, 2007), es decir, el grado de complementariedad que ejerce el aspecto presencial determina en cierta medida el éxito de las interacciones emergentes desde los ambientes de aprendizaje virtual.

\section{Recursos y herramientas utilizadas en los entornos virtuales de aprendizaje}

Las herramientas y aplicaciones que se usan como apoyo a la acción educativa y comunicativa, son derivados de la tecnología y su referente epistémico, en el cual surgen toda una serie de teorías e investigaciones que llevan a obtener una serie de desarrollos facticos e inteligibles. Dentro de este rango de aplicaciones tecnológicas, se pueden encontrar variedad de productos afianzados en diversas Instituciones de Educación Superior [IES], en la medida que potencializan el aprendizaje y coadyuvan a lograr una dinámica dialógica entre docente-estudiante. Algunas de estas consideraciones tecnológicas orientadas a la formación, se encuentran especialmente en los sistemas y plataformas -tipo LMS- que permiten la integración de elementos dinámicos proporcionados por la Web 2.0 con el fin de estimular, facilitar y consolidar un proceso formativo autónomo (De Benito y Salinas, 2005).

\section{Calidad en la educación superior}

La calidad puede definirse como un factor determinante en el nivel de educación ofrecido, a través de una serie de estándares y políticas sustentadas desde las necesidades sociales; esta calidad está asociada con la institución, docencia, investigación, entre otros factores que vinculan la acción transformadora de la institución educativa dentro de la sociedad Padilla (2008), sin embargo, el concepto de calidad abarca diversos planos de los cuales se articula la pertinencia en recursos, infraestructura y sobre todo la formación que reciben los educandos 
desde el perfil y viabilidad laboral para el desempeño efectivo en el contexto productivo.

Una de esas primeras medidas de calidad a tener en cuenta por la institución, deberá estar dirigida a la formación e inclusión del docente y sus funciones en el quehacer pedagógico e institucional, desde la profesionalización y el reconocimiento. Una organización adecuada de los directivos y coordinadores, sumado a los incentivos y procesos de formación dirigidos al docente, hacen parte de una serie de elementos que pueden contribuir a este proceso de asumir la calidad en la educación superior.

Los programas virtuales o entornos mixtos de formación, se contemplan como una medida en la cual se reforman los ambientes de aprendizaje y los medios por los cuales se orienta una educación de calidad. Algunas universidades colombianas hacen su apuesta por esta idea (Universidad de Antioquia, Universidad del Valle y Universidad Javeriana) (Facundo, 2004), utilizando el internet, la posibilidad ofrecida por las TIC y las reformas comunicativas e interactivas -Web 2.0-, persiguiendo la finalidad de establecer parámetros de excelencia para llegar a la eficiencia en términos de cobertura, satisfacción y nivel de competitividad frente a los requerimientos demandados por el entorno laboral y social; además ofrece la oportunidad de desarrollar el conocimiento "a través de una construcción colaborativa" (Fores, 2013, p.30) facilitando los procesos educativos de los sujetos en cualquiera de sus entornos

Sumado al factor tecnológicoy en relación a las variables contempladas por la institución para establecer oportunidades y servicios de calidad, uno de los aspectos más influyentes según Fernández (2009) es el desempeño del docente y la eficiencia para establecer una formación a sus estudiantes de calidad a nivel académico y personal. Para realizar un seguimiento oportuno, con el fin de establecer márgenes de calidad sobre el desempeño docente, se debe tener en cuenta un plan evaluativo configurado desde el diseño, análisis, sistematización y retroalimentación de la evaluación como un factor determinante a la hora de indagar en la labor del profesor y sus relaciones con el equipo docente y estudiantes, igualmente permitiría crear un espacio "abierto a la diversidad y a la creatividad en el aprendizaje" (Castillejo, 2015, p.440) generando nuevas propuestas pedagógicas para que el docente desempeñe su labor en ambientes dinámicos sin limitación de tiempo y espacio. 


\section{Método}

La Teoría Fundamentada o Grounded Theory es una metodología de investigación de carácter inductivo, cuyo objetivo es construir teoría que emerja desde los datos (Glaser \& Strauss, 1967; Strauss \& Corbin, 2002). Para Strauss y Corbin (2002), la teoría fundamentada puede ser utilizada, en mayor grado, en el entendimiento de un fenómeno ya estudiado para poder profundizar en él a través de las técnicas de codificación y las estrategias de análisis características de esta teoría: la codificación abierta -proceso analítico- y el método comparativo constante -cotejo de datos-.

\section{Búsqueda y selección de la información}

La búsqueda de los documentos se realizó a través de internet, gracias a la facilidad que este medio representa para el acceso y circulación de la información. En total se recopilaron y revisaron 105 documentos (Ver Gráfica 1). Los documentos seleccionados se recopilaron y guardaron como archivos digitales en los formatos PDF y RTF. En los casos donde no se podía acceder a algún documento por vía electrónica, fue necesario buscar en físico el material seleccionado y recurrir a su escaneo y digitalización con el fin de facilitar el análisis de los datos en el programa Atlas.ti.

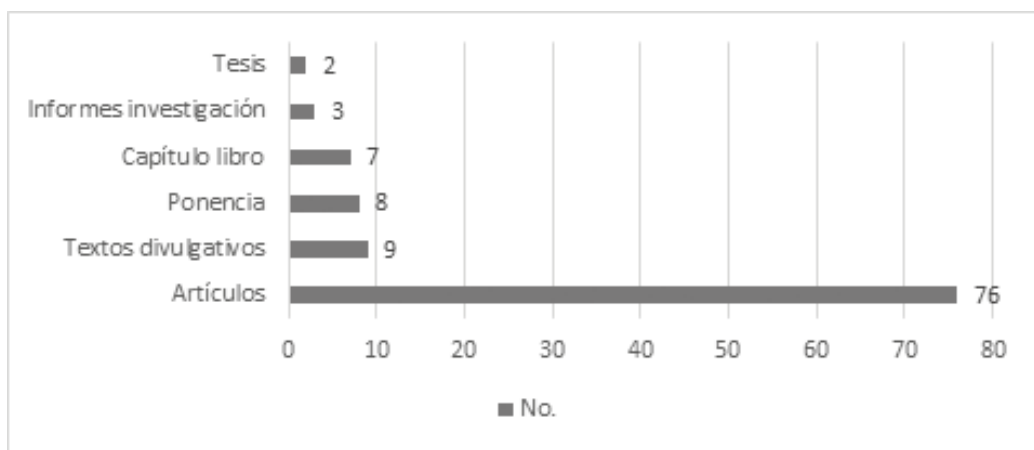

Gráfica 1. Cantidad de artículos revisados por tipo.

La mayoría de los textos revisados fueron escritos en universidades o grupos de investigación españoles. En segundo lugar, según la cantidad de documentos revisados se encuentra Colombia, seguida de Argentina y México (Ver Gráfica 2) 


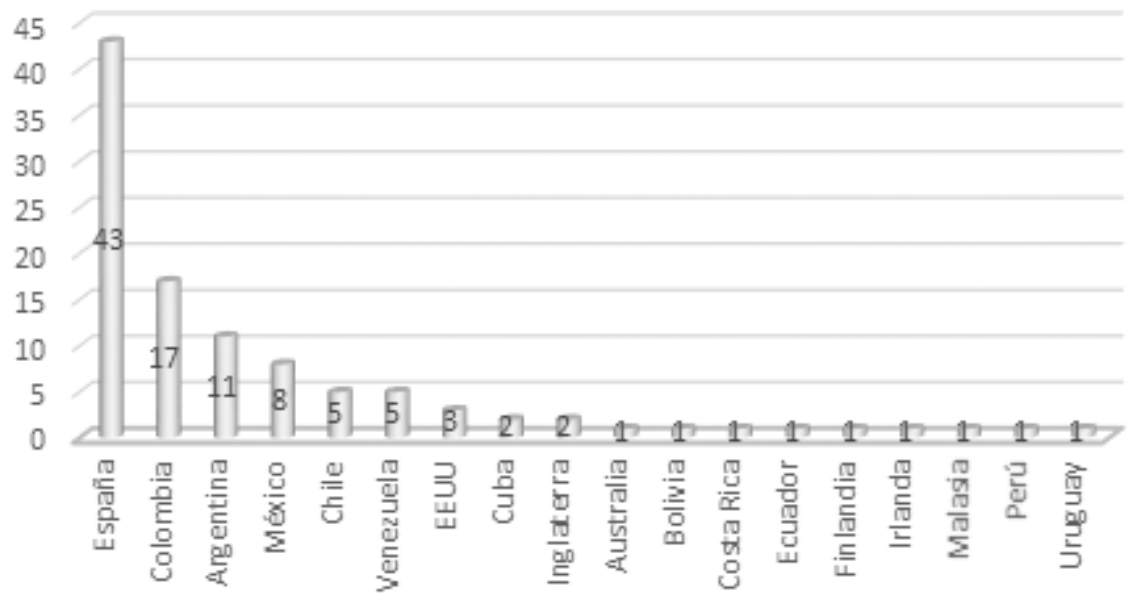

Gráfica 2. Cantidad de artículos revisados por país.

\section{Proceso de codificación}

Primera etapa: en la primera etapa se crearon códigos con el fin de realizar una clasificación inicial de los datos que permitiera reducir los documentos a unidades analizables, es decir, a fragmentos de texto con significado (citas).

Segunda etapa: en esta etapa se aplicó el método de codificación abierta para construir nuevas categorías que permitieran describir la información. Así pues, se realizó la comparación fragmento por fragmento y la asignación de las categorías necesarias que permitieran una mayor comprensión y descripción de las categorías creadas en un principio.

\section{Discusión}

El resultado del estudio, en términos reflexivos, trata de dar cuenta de la importancia de una discusión que aborda la interdependencia de las funciones del profesor, formas de evaluar en los EVA, los recursos pedagógicos y tecnológicos utilizados en estos entornos de aprendizaje, conforme su análisis proporciona un acercamiento a procesos de calidad manejados en la modalidad b-learning a nivel de educación superior. 
En un primer acercamiento a la imbricación de la calidad en el contexto $b$-learning en educación superior, se presenta la importancia de una propuesta de evaluación alterna, continua y acorde a los preceptos de calidad manejados, tanto en la administración y diseño de los EVA y las acciones que desarrolla el docente a lo largo de la formación, donde juegan un papel importante como factor de calidad. Sus acciones implican que el resto de la comunidad académica propenda por un mejoramiento en relación a su desempeño.

Tabla 1. Relación de procesos evaluativos y de calidad docente en la modalidad b-learning.

\begin{tabular}{|c|c|c|}
\hline \multicolumn{3}{|c|}{ Relación de funciones, evaluación y calidad } \\
\hline Funciones del docente & Evaluación propuesta & Ítems de calidad \\
\hline Función de planeación & *Evaluación interna & $\begin{array}{l}\text { *Calidad pedagógica } \\
{ }^{*} \text { Calidad administrativa }\end{array}$ \\
\hline Función evaluativa & ${ }^{*}$ Evaluación formativa & $\begin{array}{l}{ }^{*} \text { Calidad desempeño docente } \\
{ }^{*} \text { Calidad en formación docente }\end{array}$ \\
\hline $\begin{array}{c}\text { Función de diseño y } \\
\text { aplicación de materiales } \\
\text { educativos }\end{array}$ & $\begin{array}{l}\text { *Evaluación externa } \\
{ }^{*} \text { Evaluación interna }\end{array}$ & $\begin{array}{l}{ }^{*} \text { Calidad en material didáctico. } \\
{ }^{*} \text { Calidad docente }\end{array}$ \\
\hline Función de interacción & $\begin{array}{l}{ }^{*} \text { Evaluación diagnostica } \\
{ }^{*} \text { Evaluación interna }\end{array}$ & ${ }^{*}$ Calidad de trabajo en equipo \\
\hline Función de orientación & *Evaluación externa & $\begin{array}{l}\text { *Calidad docente. } \\
{ }^{*} \text { Calidad humanística }\end{array}$ \\
\hline Función comunicativa & $\begin{array}{l}\text { *Evaluación diagnostica } \\
\text { *Evaluación externa }\end{array}$ & $\begin{array}{c}{ }^{*} \text { Calidad de ambientes virtuales } \\
{ }^{*} \text { Calidad docente }\end{array}$ \\
\hline $\begin{array}{c}\text { Función relacionada al uso } \\
\text { de las TIC }\end{array}$ & $\begin{array}{c}{ }^{*} \text { Evaluación de entornos } \\
\text { virtuales }\end{array}$ & $\begin{array}{c}{ }^{*} \text { Calidad de programas } \\
\text { formativos } \\
{ }^{*} \text { Calidad diseño instruccional. }\end{array}$ \\
\hline
\end{tabular}

De otro lado, las perspectivas pedagógicas abordadas en el análisis e investigación del presente estudio, dieron cuenta de una relación que ya ha sido trabajada por diversos autores (De Benito y Salinas, 2003; Marcelo, 2005; Pérez y Mestre, 2007), concurrente en establecer una relación con las herramientas y medios utilizados en los ambientes virtuales de aprendizaje, por ejemplo en la perspectiva humanista: los trabajos colaborativos, los ritmos de aprendizaje individualidades y motivación, se encuentran como prioritarias en la modalidad b-learning (González, 2007). Consecuentemente, la teoría de la Gestalt, en principio, 
aporta elementos teóricos para el diseño de la interfaz y los aspectos funcionales de interacción de la plataforma (González, 2007), lo cual, en resumidas cuentas, involucra en los EVA una serie de perspectivas pedagógicas de las que pende en gran medida el diseño instruccional.

Tabla 2. Perspectivas pedagógicas y su incidencia en las funciones del docente.

\begin{tabular}{|c|c|c|}
\hline \multicolumn{3}{|c|}{ Relación perspectiva pedagógica, funciones y herramientas } \\
\hline Orientación pedagógica & Funciones de docente & Herramientas \\
\hline Constructivismo & Función de orientación & $\begin{array}{l}\text { Sesiones presenciales } \\
\text { (prácticas) }\end{array}$ \\
\hline Constructivismo social & Función de interacción & $\begin{array}{c}\text { Chat } \\
\text { Blogs } \\
\text { Redes sociales }\end{array}$ \\
\hline Aprendizaje colaborativo & Función de orientación & $\begin{array}{c}\text { Wiki } \\
\text { Foros } \\
\text { metadatos }\end{array}$ \\
\hline $\begin{array}{c}\text { Aprendizaje basado en } \\
\text { problemas }\end{array}$ & Función evaluativa & $\begin{array}{l}\text { Listas de discusiones } \\
\text { Correos electrónicos } \\
\text { Sesiones presenciales } \\
\text { (proyectos) }\end{array}$ \\
\hline Conductismo & Función comunicativa & $\begin{array}{c}\text { Módulos de refuerzo } \\
\text { FAQ } \\
\text { Tutoriales }\end{array}$ \\
\hline Enfoque en actitudes & Función de orientación & $\begin{array}{c}\text { Podcast } \\
\text { Redes sociales } \\
\text { Sesiones presenciales }\end{array}$ \\
\hline Enfoque sistémico & Función de planeación & $\begin{array}{c}\text { Glosarios } \\
\text { Calendarios compartidos } \\
\text { Web } 2.0\end{array}$ \\
\hline Cognoscitivo & Función de orientación & $\begin{array}{c}\text { Correos electrónicos } \\
\text { tutoriales }\end{array}$ \\
\hline Teoría cognitiva & $\begin{array}{c}\text { Función de aplicación de } \\
\text { material educativo }\end{array}$ & $\begin{array}{c}\text { Mapas mentales } \\
\text { Mapas conceptuales }\end{array}$ \\
\hline Humanismo & Función comunicativa & $\begin{array}{l}\text { Correos electrónicos } \\
\text { Sesionen presenciales }\end{array}$ \\
\hline Centrada en el Estudiante & $\begin{array}{l}\text { Función uso de TIC } \\
\text { Función de interacción }\end{array}$ & $\begin{array}{c}\text { Tutoriales } \\
\text { Calendarios compartidos }\end{array}$ \\
\hline Enfoque por competencias & Función de interacción & Foros de Debate \\
\hline Enfoque en Habilidades & Función de evaluación & Quiz- hot hot potatoes \\
\hline Enfoque en tecnología & Función uso de TIC & $\begin{array}{c}\text { Pantallas compartidas } \\
\text { Aulaclic } \\
\text { Metadatos }\end{array}$ \\
\hline
\end{tabular}


El estudiante y su satisfacción, penden de perfiles y roles definido con claridad, en tanto, las acciones y tareas planificadas sean realizadas en los medios presenciales y virtuales a cabalidad, bajo la responsabilidad que asume el docente con la formación en su quehacer profesional. Por consiguiente, Salinas (2008) determina la diferencia entre el rol técnico del docente y el rol profesional a través de modelos pedagógicos abiertos y cerrados enfocados para que el docente aumente su formación en habilidades pedagógicas usadas en el contexto $b$-learning.
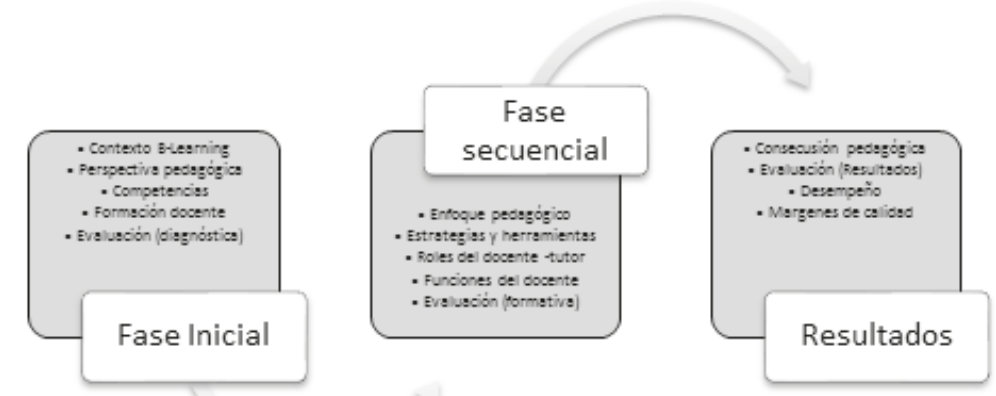

Gráfica 3. Fases de implementación de modalidades b-learning, en función del rol docente y la evaluación en dichos contextos.

Según Alegre y Villar (2007), el docente es facilitador de aprendizaje en relación a la calidad humana que ejerce e incentiva en los estudiantes por medio de la pertenencia institucional, a la par de una convicción en cuanto a un progreso formativo. De este modo, para asegurar la calidad educativa en función de las acciones realizadas por el docente (Ver Gráfica 8), bien sea en el capo presencial o en un campus virtual, se proyectan y se promueven actividades, estrategias y recursos para el uso de la información, cuya finalidad es la accesibilidad y comprensión a nivel social y cultural de las nuevas lógicas comunicativas que facilitan y determinan una acción interactiva en el proceso pedagógico de las IES.

\section{Conclusiones}

A pesar de la gran cantidad de herramientas tecnológicas, de la innumerable oferta de plataformas gratuitas y del crecimiento tecnológico en relación a su articulación con la educación, la legitimidad 
y cantidad de recursos tecnológicos no garantiza que las actividades docentes y formativas sean de calidad. El uso efectivo de estos recursos depende en buena medida del diseño pedagógico que se prevé para ellos. Desde este punto de vista, una de las competencias de los docentes debe tomar en cuenta la capacidad de incluir en los diseños pedagógicos, las herramientas y recursos tecnológicos necesarios a los fines de formación planteados por la institución, la facultad y la asignatura.

La satisfacción de los estudiantes es uno de los factores de análisis de la calidad educativa mediada con tecnologías. De esta manera, es importante la comunicación e interacción entre el docente y el estudiante para la comunicación pedagógica, a través de la realización de tutorías como funciones o competencias que los docentes en contextos $b$-Learning deben tener. Esta interpretación trata de deducir las funciones a evaluar de los docentes a partir de la respuesta a las necesidades que plantea la evaluación, es decir, a partir de la pregunta de por qué y para qué se evalúa.

El rol y funciones del docente en este contexto del b-learning, debe ser diferenciado de un anexo de actividades desempeñadas de forma virtual, pues no se trata únicamente de incluir los beneficios tecnológicos. Es más coherente hablar de un principio de reconocimiento hacia el cambio, en el cual el conocimiento y la universidad transmutan al compás de una era tecnológica en la que el docente cobra un papel protagónico como orientador y precursor de habilidades y competencias para el mundo de la vida, por ende su labor debe ser acompañada de una evaluación continua y una formación acorde a estos cambios paradigmáticos que le confieren una formación tanto pedagógica como tecnológica. 


\section{Referencias bibliográficas}

Alegre, O. y Villar, L. (2007). Modelo de Excelencia de Programas Formativos en Línea (MEPFL). Revista Interuniversitaria de Formación del Profesorado, 20 (3), 233-251.

Castillejo, D. (2015). Una mirada reflexiva a los estándares y su posible generación de ambientes virtuales de aprendizaje. Revista Interamericana de Investigación, Educación y Pedagogía. 8(2). $437-454$.

De Benito, B. y Salinas, J. (2005). Situaciones didácticas en los entornos virtuales de enseñanza aprendizaje (EVEA) en la enseñanza superior: elaboración de un instrumento de análisis. Grup de Tecnología Educativa. Palma de Mallorca: Universitat de les Illes Balears.

Fainholc, B. (2008). Modelo tecnológico en línea de Aprendizaje electrónico mixto (o Blendedlearning) para el desarrollo profesional docente de estudiantes en formación, con énfasis en el trabajo colaborativo virtual. Revista de Educación a Distancia, 21, $1-34$.

Fernández, R. (2009). Factores antecedentes en el uso de Entornos Virtuales de Formación y su efecto sobre el Desempeño Docente. Tesis Doctoral, Valencia: Universidad Politécnica de Valencia.

Fores, A. (2013). Las tic en el aprendizaje universitario, Según la edad. Revista Interamericana de Investigación, Educación y Pedagogía. 1(7).25 - 49.

Glaser, B. \& Strauss, A. (1967). The Discovery of Grounded Theory: strategies for qualitative research. New York: Aldine.

González, J. (2007). Blended learning, un modelo pertinente para la educación superior en la sociedad del conocimiento. Revista virtual EDUCA - Brasil. Disponible en: http:// e-spacio.uned.es/fez/eserv/bibliuned:19332/n07gonzamari07.pdf

González, K. y León, C. (2010). Incorporación gradual de tecnologías de la información y la comunicación en programas de educación superior. Revista Dialectica, 27, 1-13.

González, K., Padilla, J. y Rincón, D. (2011). Teorías relacionadas con el b-learning y el papel del docente. Revista Educación y desarrollo social, 5 (2), 98-111.

Gros, B. y Silva, J. (2005). La formación del profesorado como docente en los espacios virtuales de aprendizaje. Revista Iberoamericana de Educación Número, 36 (1), 1-14.

Guarneros, E., Silva, A., \& Pérez, C. (2009). La innovación educativa y tecnológica en la educación superior de México, una empresa pendiente. Cuadernos de Educación y Desarrollo, 1 (2). Disponible en: http://reposital.cuaed.unam.mx:8080/jspui/ handle/123456789/1087 
Marcelo, C. (2005). Evaluación en e-learning: Compartiendo algunas experiencias. Sevilla: Universidad de Sevilla.

Padilla, J. (2008). Globalización y Educación superior: Un reto en la formación del docente universitario. Bogotá: Editorial Bonaventuriana.

Pérez, R. y Mestre, U. (2007). Monografía sobre b-Learning o aprendizaje Bimodal. La Habana: Editorial Universitaria.

Regnet, M. y Martínez, D. (2010). Desarrollo de una Aplicación de b-Learning para el Aprendizaje del AutonomicComputin. Tesis de Licenciatura, Corrientes: Universidad Nacional del Nordeste.

Rojas Mesa, J., \& Leal Urueña, L. (2014). Entre flujos y fronteras: la educación superior mediada tecnológicamente vista a través de una perspectiva etnográfica. Revista De Investigaciones UNAD, 13(2), 9-27. doi:http://dx.doi.org/10.22490/25391887.1143

Salinas, J. (2008). Modelos didácticos en los campus virtuales universitarios: Patrones metodológicos generados por los profesores en procesos de enseñanza-aprendizaje en entornos virtuales. Palma de Mallorca: Universitat de les Illes Balears.

Sánchez, J. (2008). Una experiencia docente basada en el "blendedlearning" en métodos de investigación educativa. Universidad de Castilla-La Mancha. Disponible en: http://www.uem.es/myfiles/pageposts/jiu/jiu2010/pdf/107c.pdf

Strauss, A. y Corbin, J. (2002). Bases de la investigación cualitativa. Técnicas y procedimientos para desarrollar la teoría fundamentada. Medellín: Editorial Universidad de Antioquia. 